\title{
XPC Promotes Pluripotency of Human Dental Pulp Cells through Regulation of Oct-4/Sox2/c-Myc
}

\author{
Lu Liu, Zhengjun Peng, Zhezhen Xu, and Xi Wei \\ Operative Dentistry and Endodontics, Guanghua School of Stomatology, Affiliated Stomatological Hospital, \\ Guangdong Province Key Laboratory of Stomatology, Sun Yat-sen University, Guangzhou, Guangdong 510055, China
}

Correspondence should be addressed to Xi Wei; weixi@mail.sysu.edu.cn

Received 24 January 2016; Revised 8 March 2016; Accepted 13 March 2016

Academic Editor: Boon C. Heng

Copyright (C) $2016 \mathrm{Lu}$ Liu et al. This is an open access article distributed under the Creative Commons Attribution License, which permits unrestricted use, distribution, and reproduction in any medium, provided the original work is properly cited.

Introduction. Xeroderma pigmentosum group C (XPC), essential component of multisubunit stem cell coactivator complex (SCC), functions as the critical factor modulating pluripotency and genome integrity through interaction with Oct-4/Sox 2 . However, its specific role in regulating pluripotency and multilineage differentiation of human dental pulp cells (DPCs) remains unknown. Methods. To elucidate the functional role XPC played in pluripotency and multilineage differentiation of DPCs, expressions of XPC in DPCs with long-term culture were examined by real-time PCR and western blot. DPCs were transfected with lentiviral-mediated human XPC gene; then transfection rate was investigated by real-time PCR and western blot. Cell cycle, apoptosis, proliferation, senescence, multilineage differentiation, and expression of Oct-4/Sox $2 / \mathrm{c}-\mathrm{Myc}$ in transfected DPCs were examined. Results. XPC, Oct-4, Sox2, and c-Myc were downregulated at P7 compared with P3 in DPCs with long-term culture. XPC genes were upregulated in DPCs at P2 after transfection and maintained high expression level at P3 and P7. Cell proliferation, PI value, and telomerase activity were enhanced, whereas apoptosis was suppressed in transfected DPCs. Oct-4/Sox 2/c-Myc were significantly upregulated, and multilineage differentiation in DPCs with XPC overexpression was enhanced after transfection. Conclusions. XPC plays an essential role in the modulation of pluripotency and multilineage differentiation of DPCs through regulation of Oct-4/Sox $2 / \mathrm{c}-\mathrm{Myc}$.

\section{Introduction}

Dental pulp cells (DPCs), characterized by self-renewal, multilineage differentiation capability, and easy accessibility, are regarded as potential cell source for dental engineering [1]. Despite potential application in dental tissue regeneration, DPCs have been used for various degenerative diseases such as Alzheimer's disease, myocardial infarction, diabetes mellitus, bone defects, and spinal cord injuries [2]. However, it has been demonstrated that the progenitor/stem cell population was limited in DPCs, and primary cells with longterm in vitro culture were known to undergo replicative senescence, which limited pluripotency and restricted the potential application of DPCs in dental bioengineering [3]. Therefore, an investigation of the molecular mechanism to elevate the pluripotency of DPCs and optimize the cell characteristics would be of critical significance.
Previous studies revealed that overexpression of Oct-4, Sox2, Klf4, and c-Myc (OSKM) could induce mouse fibroblasts into induced pluripotent stem cells (iPSCs) with similar gene expression and developmental potential of mouse embryonic stem cells (mESCs) [4]. The endogenous expression level of Oct-4, Sox2, and c-Myc has been confirmed to be correlated with reprogramming and the pluripotency [4]. Oct- 4 and Sox2, locating at the central of the transcriptional network, work cooperatively in maintaining pluripotency and inducing the reprogramming [5]. Recent study reported that a multisubunit stem cell coactivator complex (SCC) is required to activate the transcription of Oct-4/Sox 2 and downstream target genes in ESCs. SCC is composed of the nucleotide excision repair (NER) proteins including xeroderma pigmentosum group C (XPC), RAD23B, and CETN2 [6]. XPC, as one component of the NER, serves as a transcriptional coactivator of DNA repair through 
TABLE 1: Primer sequences used in quantitative real-time polymerase chain reaction.

\begin{tabular}{|c|c|}
\hline Gene & Primers \\
\hline \multirow{2}{*}{ XPC } & Forward: $5^{\prime}$-TGAGACCATACCAGAGCCCA-3' \\
\hline & Reverse: $5^{\prime}$-GGGCATACAGAGGGTGGTTC-3' \\
\hline \multirow{2}{*}{ Oct- 4} & Forward: 5'-GCT CGA GAA GGA TGT GGT C-3' \\
\hline & Reverse: $5^{\prime}$-ATC CTC TCG TTG TGC ATA GTC G-3' \\
\hline \multirow{2}{*}{ Sox 2} & Forward: 5'-GAGAACCCCAAGATGCACAAC-3' \\
\hline & Reverse: $5^{\prime}$-CGCTTAGCCTCGTCGATGA-3' \\
\hline \multirow{2}{*}{ c-Myc } & Forward: 5'-GGCTCCTGGCAAAAGGTCA-3' \\
\hline & Reverse: 5'-AGTTGTGCTGATGTGTGGAGA-3' \\
\hline \multirow{2}{*}{$18 \mathrm{~s}$} & Forward: 5'-CCTGGATACCGCAGCTAGGA-3' \\
\hline & Reverse: $5^{\prime}$-GCGGCGCAATACGAATGCCCC-3' \\
\hline
\end{tabular}

modifying chromatin. XPC functions as critical factor modulating pluripotency, reprogramming, and genome integrity in mESCs through interacting with Oct-4 [7]. Lacking of XPC or RAD23B caused significant decrease of reprogramming to iPSCs, implying that the expression level of XPC may be related to the establishment efficiency of iPSCs [6].

In our previous study, we demonstrated that Oct-4, Sox2, and c-Myc were downregulated in DPCs with long-term in vitro culture, indicating that Oct- 4 was closely related to the pluripotency of DPCs [8]. Considering that XPC interacts directly with Oct- 4 and functions as critical factor modulating pluripotency, we established the XPC overexpression DPCs model by lentivirus transfection and extended our study to investigate the modulatory role of XPC played in cell proliferation and apoptosis and cell cycle and senescence and expression of pluripotency markers (Oct-4, Sox2, and c-Myc) and multilineage differentiation capability of DPCs during long-term in vitro culture. The data generated from this study will shed light on elevating the pluripotency of DPCs and their application in dental engineering.

\section{Materials and Methods}

2.1. Isolation and Expansion of Human DPCs. Normal human premolars and impact third molars were extracted from healthy young adults (12-28 years) undergoing orthodontic treatment at the Department of Oral and Maxillofacial Surgery, the Affiliated Stomatology Hospital of Sun Yatsen University; informed consent was obtained from each patient. The protocol was approved by the University Ethic Committee. DPCs were obtained from human dental pulp tissues by explant culture as previously described [3]. DPCs were cultured in Dulbecco's modified Eagle medium with low glucose (DMEM-LG, Invitrogen, VIC, Australia) supplemented with $10 \%$ fetal bovine serum (FBS, HyClone, UT, USA), $10 \mathrm{U} / \mathrm{mL}$ penicillin $\mathrm{G}$, and $10 \mathrm{mg} / \mathrm{mL}$ streptomycin (Invitrogen, VIC, Australia). DPCs were incubated at $37^{\circ} \mathrm{C}$ in $5 \% \mathrm{CO}_{2}$. The medium was changed every 3 days.

\subsection{Quantitative Real-Time Reverse Transcription-Polymerase} Chain Reaction for the Expression of XPC, Oct-4, Sox2, and $c-M y c$ in DPCs. Expression of XPC in DPCs from P1 to P7 was examined by real-time RT-PCR as previously described
[3], while only the representative results of P3 and P7 were presented in Results. Total RNA of DPCs was isolated using Trizol reagent (Invitrogen, NY, USA) following the manufacturer's protocol. The concentration and quality of RNA samples were measured with spectrophotometers and gel electrophoresis. The first-strand cDNA was synthesized from $1 \mathrm{mg}$ of total RNA using SuperScript III (Invitrogen, NY, USA) in a total volume of $20 \mathrm{~mL}$. About $2.5 \mathrm{~mL}$ of the reaction mixture was incubated with SYBR Green I Master Mix (Applied Biosystems, NY, USA) in a total volume of $25 \mathrm{~mL}$. The conditions for polymerase chain reaction (PCR) were as follows: $95^{\circ} \mathrm{C}$ for $10 \mathrm{~min}$ for activation, followed by 40 cycles of denaturation at $95^{\circ} \mathrm{C}$ for $15 \mathrm{~s}$ each, and, finally, primer extension at $60^{\circ} \mathrm{C}$ for $1 \mathrm{~min}$. XPC, Oct-4, Sox2, c-Myc, and $18 s$ mRNA were quantified using ABI Prism 7000 sequence detection system (Applied Biosystems, NY, USA). Each plate contained $18 s$ as the housekeeping gene to normalize the PCR data. Primers used for detection were listed in Table 1. All experiments were repeated triplicate. Raw data were acquired to calculate the threshold cycle $(\mathrm{Ct})$ value and relative gene expression values. The delta-delta $\mathrm{Ct}$ method was performed to analyze the results.

2.3. Western Blot for the Expression of XPC, Oct-4, Sox2, and $c-M y c$ in DPCs. Western blot was performed as described previously [9]. Briefly, total protein of DPCs at P1 to P7 was measured by Bio-Rad Coomassie Blue protein assay (BioRad Laboratories, Richmond, CA, USA), whereas only the representative results of P3 and P7 were presented in Results. Twenty micrograms of protein was diluted by $10 \%$ bromophenol blue and boiled before being separated by sodium dodecyl sulfate-polyacrylamide gel electrophoresis (SDS-PAGE) and transferred to a nitrocellulose membrane. The membranes were blocked in 5\% low-fat milk at room temperature for $1 \mathrm{~h}$, rinsed, and incubated with monoclonal antibodies mouse against human XPC (1:500 dilution, Abcam, MA, USA), Oct4 (1:100, Chemicon, MA, USA), Sox2 (1:50, R\&D System, MN, USA), c-Myc (1:50, Santa Cruz, CA, USA), or human GAPDH (1:1000 dilution, Santa Cruz, CA, USA) overnight at $4^{\circ} \mathrm{C}$. After washing, the membrane was incubated with the HRP-conjugated secondary antibody (1:5000 dilution, Jackson, USA) at room temperature for $1 \mathrm{~h}$. Immunoreactive proteins were then visualized by incubating membranes with 
electrogenerated chemiluminescence plus detection agents (GE Healthcare, USA).

2.4. Lentivirus Transfection of XPC in DPCs. The CDS region of XPC gene was amplified from a plasmid containing the full-length XPC sequence and cloned into Lentivector. After transformation into competent $E$. coli, the candidate clones were identified by PCR and sequencing. The constructed recombinant shuttle plasmid and lentivirus helper plasmid were cotransfected into HEK293T cells with the mediation of Lipofectamine 2000 and then propagated. A replicationdeficient Lentivector, carrying the human XPC gene and the reporter gene, EGFP gene, was used in the current study. Forty-eight hours after transfection, EGFP expression in DPCs at passage 2 was observed by a fluorescence microscope (Axiovert, Zeiss, Germany).

2.5. Quantitative Real-Time PCR and Western Blot for Evaluation of XPC Expression in DPCs after Transfection. The expression of XPC in DPCs with XPC overexpression at P2, P3, and P7 was examined using real-time PCR and western blot $48 \mathrm{~h}$ after transfection as mentioned above. The nontransfected cells and cells transfected with vector served as control groups. Briefly, quantification of XPC and $18 s$ mRNA was performed using ABI Prism 7000 sequence detection system (Applied Biosystems, NY, US). Primers used for detection were listed in Table 1. Delta-delta Ct method was performed to analyze the result. The total protein was measured by a Bio-Rad Coomassie Blue protein assay (Bio-Rad Laboratories, Richmond, CA, US). Proteins were detected with mouse monoclonal antibodies against human XPC (1:500, Abcam, MA, US) and GAPDH (1:1000 dilution, Santa Cruz Biotechnology, CA, US).

2.6. Cell Counting Kit 8 (CCK8) and Telomerase Activity Assay for Cell Proliferation and Senescence of DPCs with XPC Overexpression. Cell proliferation was measured by Cell Counting Kit 8 (CCK8) assay. DPCs of XPC overexpression group, vector group, and nontransfected group were grown in 96well plates. The cells were cultured in DMEM supplemented with $10 \%$ FCS and $50 \mathrm{mg} / \mathrm{mL}$ gentamycin. Cell proliferation of DPCs at passages 3 and 7 at different time points $(0,3 \mathrm{~d}, 5 \mathrm{~d}$, $7 \mathrm{~d}$ ) was evaluated using the CCK8 (Dojindo, Tokyo, Japan), while only the representative results of $5 \mathrm{~d}$ were presented. $10 \mu \mathrm{L}$ of CCK 8 solution was added to the culture medium and incubated for additional $3 \mathrm{~h}$. The absorbance was determined at $450 \mathrm{~nm}$ wave length. Telomerase activity in DPCs from passages DPCs from passages 3 and 7 with/without XPC overexpression was detected using a quantitative telomerase detection kit (Allied Biotech, MD, US).

2.7. Flow Cytometry for Cell Cycle and Apoptosis. The cultures of DPCs were serum deprived for $24 \mathrm{~h} .1 \times 10^{5}$ DPCs of XPC overexpression group, vector group, and nontransfected group were harvested by trypsinization, washed twice in cold PBS, and fixed in $70 \%$ alcohol for $30 \mathrm{~min}$ on ice. After washing in cold PBS 3 times, cells were incubated with $0.5 \%$ propidium iodinate (PI) for 30 minutes at $4^{\circ} \mathrm{C}$. Cells were analyzed using
FACSCalibur flow cytometer (BD Biosciences, San Jose, CA, US). Data was analyzed using FCSExpress software.

2.8. Effect of XPC Overexpression on the Expression of Pluripotent Markers in DPCs. The expression of Oct-4, Sox2, and $\mathrm{c}-\mathrm{Myc}$ on DPCs with XPC overexpression was investigated by immunofluorescent staining and real-time PCR. DPCs at P3 and P7 were cultured in chamber slides (Nunc, NY, USA) until $80 \%$ confluence and fixed with 3\% paraformaldehyde for $15 \mathrm{~min}$. The slides were washed in PBS 3 times, permeabilized with $0.1 \%$ Triton for $20 \mathrm{~min}$, and incubated with $10 \%$ swine serum for $1 \mathrm{~h}$. Slides were transferred to a humidified chamber and stained with XPC (1:400 dilution, Abcam, MA, USA), Oct-4 (1:400 dilution, Chemicon, MA, USA), Sox2 ( $1: 400$ dilution, R\&D System, MN, USA), and c-Myc ( $1: 400$ dilution, Santa Cruz, CA, USA) antibodies overnight at $4^{\circ} \mathrm{C}$. PBS was used as control. Samples were washed 3 times in PBS and incubated with a fluorochrome-labeled secondary antibody ( $1: 100$ dilution, Invitrogen, NY, USA) for $3 \mathrm{~h}$. The sections were thoroughly washed in PBS and mounted for fluorescent microscopic analysis (Axiovert, Zeiss, Germany). Real-time PCR was performed as mentioned above. Total RNA of DPCs at P3 and P7 was isolated by Trizol reagent. Primers used for detection were listed in Table 1. Each plate contained $18 s$ as the housekeeping gene to normalize the PCR data. Delta-delta Ct method was performed to analyze the result.

2.9. Effect of XPC Overexpression on Multilineage Differentiation of DPCs. For odontogenic differentiation, DPCs of XPC overexpression group, vector group, and nontransfected group were odontogenically induced in growth medium containing $10 \mathrm{mM} \beta$-glycerophosphate (Sigma-Aldrich, Australia), $50 \mu \mathrm{M}$ ascorbic acid (Sigma-Aldrich, Australia), and $100 \mathrm{nM}$ dexamethasone (Sigma-Aldrich, Australia) for $21 \mathrm{~d}$. The expression of DSPP was explored by immunofluorescent staining as mentioned above. DPCs were stained with DSPP antibody (1:400 dilution, Chemicon, MA, USA). The mRNA expression of DMP1 and DSPP was evaluated by real-time PCR. Primers used for detection are listed in Table 2.

For adipogenic differentiation, DPCs were incubated in the adipogenic induction medium consisting of $0.5 \mathrm{mM} \mathrm{3-}$ isobutyl-1-methylxanthine (IBMX, Sigma, USA), $10 \mu \mathrm{g} / \mathrm{mL}$ insulin (GIBCO, USA), $1 \mathrm{mM}$ dexamethasone, $100 \mu \mathrm{M}$ indomethacin (Sigma, USA), and 15\% FBS in $\alpha$-MEM for $3 \mathrm{~d}$, followed by the adipogenic maintenance medium consisting solely of $10 \mu \mathrm{g} / \mathrm{mL}$ insulin and 15\% FBS for $1 \mathrm{~d}$. After completing the three cycles of induction and maintenance, the induced cells were incubated for another $14 \mathrm{~d}$ in adipogenic maintenance medium. Adipogenic differentiation was confirmed by immunofluorescent staining and the mRNA expression of LPL and PPAR 22 was confirmed by real-time PCR. DPCs were stained with LPL antibody (1:400 dilution, Chemicon, MA, USA).

For chondrogenic differentiation, DPCs were chondrogenically inducted by culturing in high cell density through pelletation $\left(2 \times 10^{5}\right.$ cells per pellet $)$ in $500 \mu \mathrm{L}$ chondrogenic differentiation medium. Serum-free chondrogenic differentiation medium consisted of high glucose DMEM 
TABLE 2: Primer sequences used in quantitative real-time polymerase chain reaction.

\begin{tabular}{|c|c|}
\hline Gene & Primers \\
\hline \multirow{2}{*}{ DMP1 } & Forward: $5^{\prime}$-CTGCAACACAGG GAAATGGA-3' \\
\hline & Reverse: $5^{\prime}$-ACGGACACTGCTC CATCCTT-3' \\
\hline \multirow{2}{*}{ DSPP } & Forward: 5'-ATT CCG GTT CCC CAG TTA GTA-3' \\
\hline & Reverse: $5^{\prime}$-CTG TTG CTA GTG GTG CTG TT-3' \\
\hline \multirow{2}{*}{$\operatorname{PPAR} \gamma 2$} & Forward: $5^{\prime}$-CTT CGG AAT CAG CTC TGT GGA C-3' \\
\hline & Reverse: $5^{\prime}$-GCA TCC TTC ACA AGC ATG GAC T-3' \\
\hline \multirow{2}{*}{ LPL } & Forward: $5^{\prime}$-GGG AGT TTG GCT CCA GAG TTT-3' \\
\hline & Reverse: $5^{\prime}$-TGT GTC TTC AGG GGT CCT TAG-3' \\
\hline \multirow{2}{*}{ Col2al } & Forward: 5'-TCC CAG AAC ATC ACC TAC CAC T-3' \\
\hline & Reverse: $5^{\prime}$-GGT CTT CTG TGA TCG GTA CTC G-3' \\
\hline \multirow{2}{*}{$18 \mathrm{~s}$} & Forward: 5'-CCTGGATACCGCAGCTAGGA-3' \\
\hline & Reverse: 5' -GCGGCGCAATACGAATGCCCC-3' \\
\hline
\end{tabular}

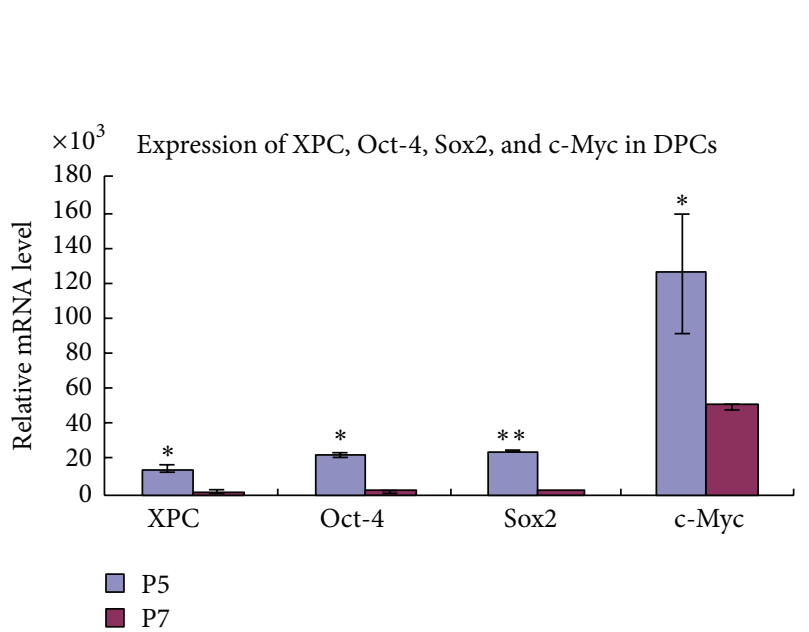

(a)

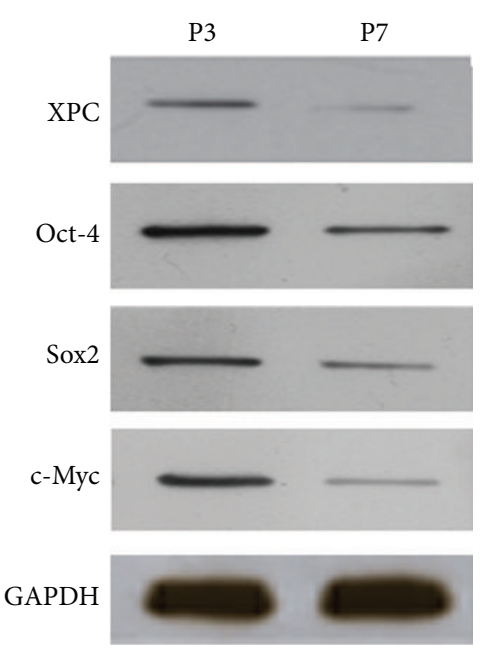

(b)

FIGURE 1: Expression of XPC, Oct-4, Sox2, and $c$-Myc in DPCs at various passages. Real-time PCR showed that mRNA expression of XPC, Oct4 , Sox2, and $c-M y c$ was significantly higher in DPCs at passage 3 compared with passage 7 (a) $\left({ }^{*} p<0.05,{ }^{* *} p<0.001\right)$. Western blot showed that the protein expression of XPC, Oct-4, Sox2, and c-Myc revealed similar expression pattern with real-time PCR, which was stronger in DPCs at passage 3 compared with passage 7 (b).

supplemented with $10 \mathrm{ng} / \mathrm{mL}$ of transforming growth factor$\beta 3$ (TGF- $\beta 3$, R\&D Systems, USA), $10 \mathrm{nM}$ dexamethasone, $50 \mathrm{mg} / \mathrm{mL}$ of ascorbic acid, $10 \mathrm{mg} / \mathrm{mL}$ of sodium pyruvate (Sigma, USA), $10 \mathrm{mg} / \mathrm{mL}$ of proline (Sigma, USA), and an insulin-transferrin-selenium supplement. Pellets were allowed to differentiate under 3-dimensional conditions in $15 \mathrm{~mL}$ centrifuge tubes at $2 \%$ or $20 \% \mathrm{O}_{2}$ tension. After $21 \mathrm{~d}$ of chondrogenic differentiation, the pellets were fixed with $4 \%$ PFA and embedded in paraffin. Expression of collagen type II was examined by immunofluorescent staining and realtime PCR. DPCs were stained with collagen type II antibody (1:400 dilution, Chemicon, MA, USA).

2.10. Statistical Analysis. All experiments were repeated at least three times. The SPSS19.0 software package (SPSS Inc., Chicago, IL) was used for the statistical tests. All the data were analyzed using one-way ANOVA analysis and Student's $t$-test. The difference was considered as being of statistical significance at $p<0.05$.

\section{Results}

3.1. Expression of XPC, Oct-4, Sox2, and c-Myc in DPCs at Various Passages. Expression of XPC in DPCs from passages 1 to 7 were investigated by real-time PCR and western blot, while only the results of representative passages (P3 and P7) were presented. Real-time PCR (Figure 1(a)) and western blot (Figure 1(b)) indicated that the mRNA and protein expression of XPC, Oct-4, Sox2, and c-Myc showed similar expression pattern, which was significantly downregulated at P7 compared with P3 $\left({ }^{*} p<0.05,{ }^{* *} p<0.001\right)$.

3.2. Gene Transfection and Expression of XPC in DPCs. Expression of $\mathrm{pCDH}-\mathrm{CMV}-\mathrm{XPC}-\mathrm{EF} 1-\mathrm{copGFP}$ plasmid in 


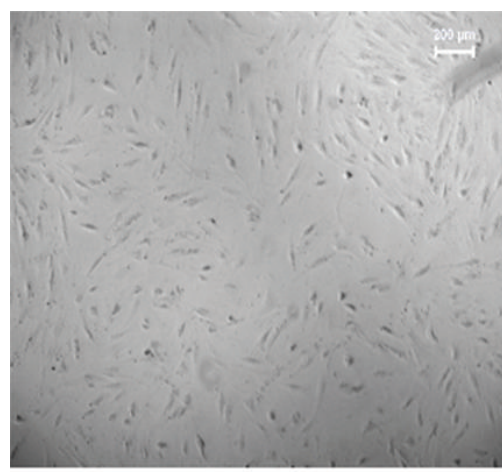

(a1)

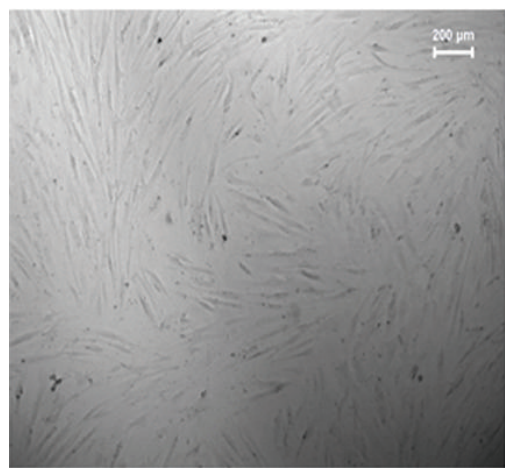

(a4)

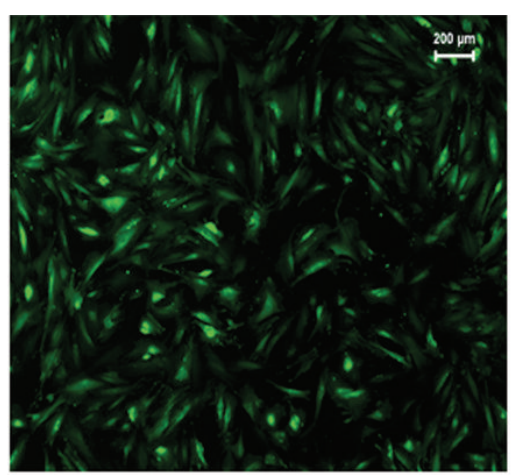

(a2)

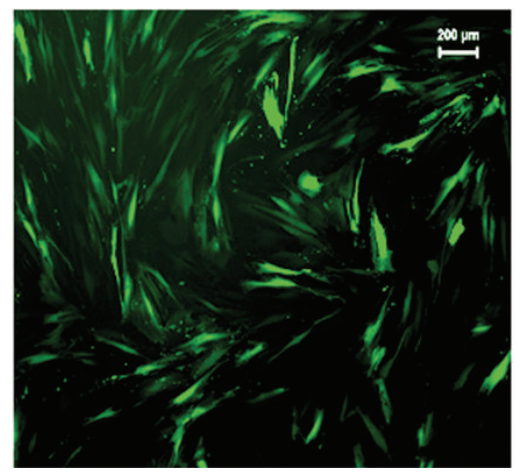

(a5)

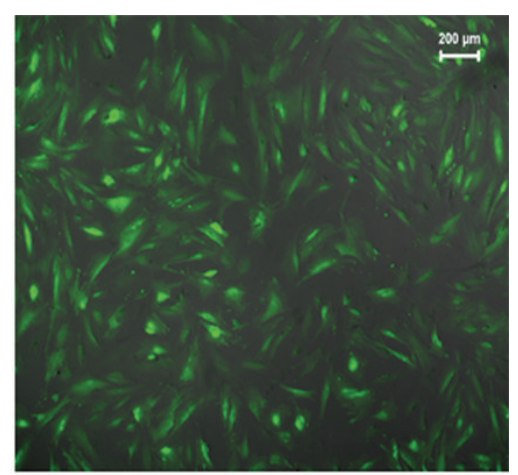

(a3)

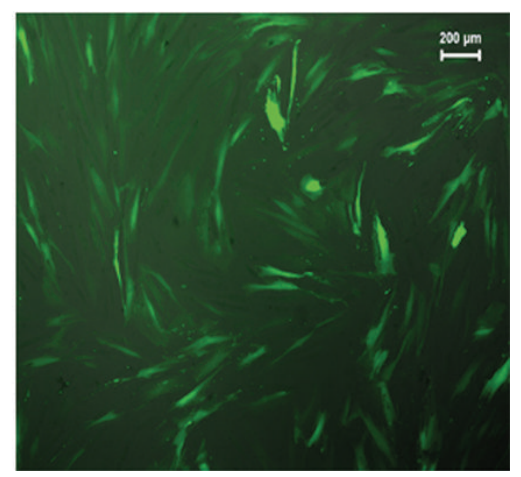

(a6)

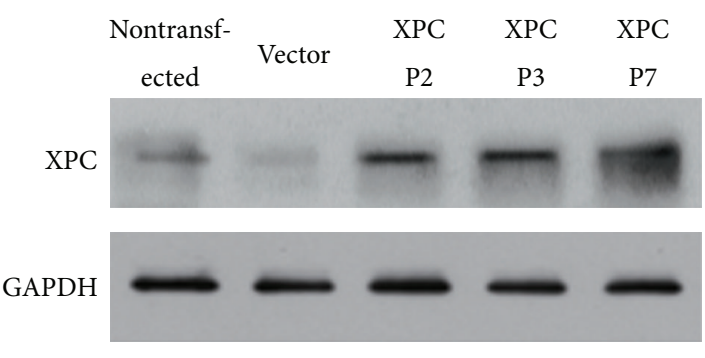

(b1)

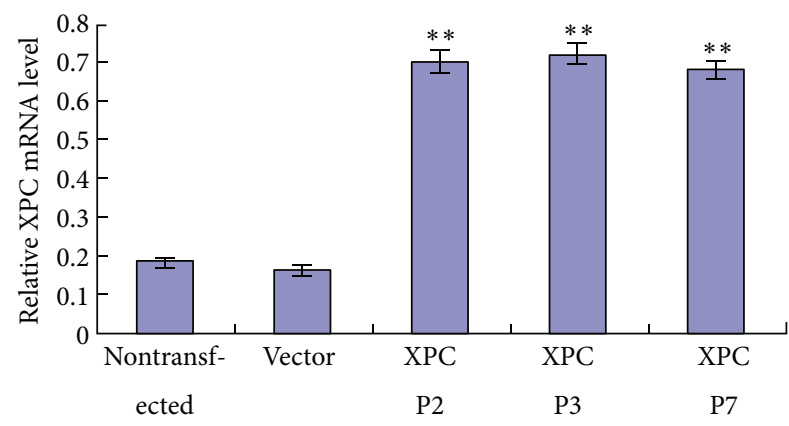

(b2)

FIGURE 2: Establishment of XPC overexpression DPCs model. Expression of pCDH-CMV-XPC-EF1-copGFP plasmid in HEK293T cells and DPCs at P2 (a1-a6). Strong green fluorescence was detected in HEK293T cells $(\mathrm{a} 2, \times 50)$ and DPCs $(\mathrm{a} 5, \times 100)$ after transfection. Combined figures of the bright field and fluorescence figure (a3, a6). Western blot and real-time PCR showed that the expression of XPC was enhanced in XPC+/DPCs at P2 at both protein and mRNA level compared with vector group, and XPC maintained high expression level in DPCs at P3 and P7 $\left({ }^{* *} p<0.01\right)(\mathrm{b} 1, \mathrm{~b} 2)$.

HEK293T cells and DPCs was shown in Figure 2. Strong green fluorescence could be viewed in HEK293T cells after transfection (Figure 2(a2)). All DPCs with XPC overexpression showed green fluorescent staining (Figure 2(a5)). The result of real-time PCR revealed that XPC mRNA was significantly higher in XPC transfected DPCs at P2, P3, and P7 compared with the vector group (Figure 2 (b2), ${ }^{* *} p<$ $0.001)$, whereas there is no significant difference between nontransfected and vector groups $(p>0.05)$. Western blot (Figure 2(b1)) demonstrated that the protein expression of XPC was strongly upregulated in DPCs at P2, P3, and P7 after transfection, which agreed with the result of real-time PCR (Figure 2(b2)). These results indicated that the XPC overexpression DPCs model was successfully established, which could be passaged and XPC maintained consistently high expression level in transfected DPCs even after longterm in vitro culture.

3.3. Cell Proliferation, Cycle, and Apoptosis of DPCs with $X P C O$ verexpression. To investigate the biological function of XPC in DPCs, CCK8 and FACS were applied to DPCs with $\mathrm{XPC}$ overexpression. CCK8 revealed that cell proliferation 
TABLE 3: Cell cycle of DPCs with long-term in vitro culture after XPC transfection.

\begin{tabular}{lcccc}
\hline Groups & G0/G1 & G2/M & S & PI $=(\mathrm{G} 2 / \mathrm{M}+\mathrm{S}) \%$ \\
\hline Nontransfected P3 & $87.1 \pm 1.5$ & $8.04 \pm 1.4$ & $4.76 \pm 1.5$ & $12.8 \pm 1.5$ \\
Vector P3 & $87.6 \pm 1.6$ & $7.92 \pm 1.5$ & $4.28 \pm 1.5$ & $12.3 \pm 1.6$ \\
XPC P3 & $85.2 \pm 1.1^{*}$ & $8.97 \pm 1.5^{*}$ & $5.73 \pm 1.5^{*}$ & $14.7 \pm 1.1^{*}$ \\
Nontransfected P7 & $95.8 \pm 0.4$ & $2.04 \pm 0.4$ & $2.05 \pm 0.4$ & $4.09 \pm 0.4$ \\
Vector P7 & $95.9 \pm 0.4$ & $2.01 \pm 0.4$ & $2.03 \pm 0.4$ & $4.04 \pm 0.4$ \\
XPC P7 & $94.9 \pm 0.4^{*}$ & $3.12 \pm 0.4^{*}$ & $1.90 \pm 0.4^{*}$ & $5.02 \pm 0.4^{*}$ \\
\hline
\end{tabular}

$($ Mean $\pm \mathrm{SD} \% N=3)$.

${ }^{*} P<0.05$.

of transfected DPCs at both P3 and P7 was significantly enhanced (Figure $3(\mathrm{bl}),{ }^{* *} p<0.001$ ), whereas there is no significant difference between control group and vector group $(p>0.05)$. The result of FACS demonstrated that the cell number of transfected DPCs at P3 and P7 in G0/G1 was significantly downregulated compared with the vector groups; DPCs were arrested in G2/M and S phase. The percentage of $\mathrm{PI}=(\mathrm{S}+\mathrm{G} 2 / \mathrm{M})$ value and telomerase activity of transfected DPCs at both P3 and P7 was significantly upregulated, albeit the apoptosis rate was significantly downregulated (Figures 3(b2)-3(b4), Table 3, p < 0.05). Therefore, XPC could enhance cell proliferation and telomerase activity of DPCs, arrested cells in G2/M and S phase, and upregulated PI value, albeit suppressed apoptosis, suggesting the role XPC played in the maintenance of pluripotency in DPCs.

3.4. Expression of Oct-4, Sox2, and c-Myc in DPCs with XPC Overexpression. Real-time PCR indicated that the mRNA level of Oct-4, Sox2, and $c-M y c$ was significantly upregulated in DPCs with XPC transfection at both P3 and P7 (Figures $\left.4(\mathrm{~b} 1)-4(\mathrm{~b} 3),{ }^{* *} p<0.001\right)$. Similarly, immunofluorescent staining revealed that XPC (Figure 4(a1)), Oct-4 (Figure 4(a2)), Sox2 (Figure 4(a3)), and c-Myc (Figure 4(a4)) were strongly expressed in DPCs with XPC overexpression at P7, mainly located in nucleus and moderately expressed in cytoplasm. Whereas XPC (Figure 4(a5)), Oct-4 (Figure 4(a6)), Sox2 (Figure 4(a7)), and c-Myc (Figure 4(a8)) were barely detected in DPCs at P7 without transfection. Oct4, Sox2, and c-Myc showed extensive expression in DPCs at P3 with/without XPC expression (data not shown).

3.5. The Multilineage Differentiation of DPCs with XPC Overexpression. The odontogenic/adipogenic/chondrogenic differentiation capability of DPCs with XPC overexpression at P7 was investigated by immunofluorescent staining (Figures 5(a1)-5(a6)), and the mRNA expression of lineage related genes in DPCs at P3 and P7 was further confirmed by real-time PCR. After odontogenic/adipogenic/chondrogenic induction, immunofluorescent staining indicated that DSPP (Figure 5(a1)), LPL (Figure 5(a2)), and Collagen type II (Figure 5(a3)) were extensively expressed in DPCs at P7 with XPC overexpression, strongly expressed in the nucleus and moderately expressed in cytoplasm. However, the expression of DSPP (Figure 5(a4)), LPL (Figure 5(a5)), and Collagen type II (Figure 5(a6)) was barely detected in the DPCs at
P7 without XPC transfection. Real-time PCR revealed that odontogenic markers ( $D M P 1, D S P P)$, adipogenic markers (LPL, PPAR 2 2), and chondrogenic marker (Collagen type II) showed similar expression pattern, upregulated in DPCs with XPC overexpression at both P3 and P7 compared with the vector groups (Figures 5(b1)-5(b5), ${ }^{*} p<0.05,{ }^{* *} p<0.01$ ). Therefore, XPC could effectively enhance the multilineage differentiation capability of DPCs with long-term in vitro culture.

\section{Discussion}

Somatic cells could be reprogrammed to iPSCs with ESCslike characteristics through introduction of Oct-4, Sox2, cMyc, Klf4, and Nanog [4]. Oct-4 is one of the key factors regulating pluripotency and reprogramming. Oct- 4 expression at the optimized level is necessary for self-renewal of mESCs [10]. The identification of Oct-Sox 2 composite consensus sequence confirmed that Oct- 4 activates transcription of the pluripotency-associated transcription factors (TFs) in the heterodimer through direct cooperation with Sox2. Oct4/Sox 2 complexes are key transcriptional activators to recognize the specific DNA cis-acting elements in reprogramming markers [5]. The Oct-Sox2 cis-control element is a common configuration in the promoters of Oct-4/Sox 2 -activated genes, through which Oct- 4 and Sox 2 cooperate with various cofactors to activate the pluripotency network and initiate the reprogramming process [11, 12]. Although Oct-4-Sox 2 complexes are at the top of the regulatory hierarchy, some studies indicated that even the coexpression of Oct- 4 and Sox 2 was not sufficient to activate the Nanog promoter reporter in 293 or NIH3T3 cells [12]. Recent study reported that SCC is required to activate the transcription of Oct- $4 / \operatorname{Sox} 2$ and downstream target genes in ESCs. SCC could maintain and reestablish stem cell pluripotency through functioning as a transcriptional coactivator of Oct-4/Sox 2 . SCC interacts directly with Oct-4/Sox 2 and is recruited to the Oct- 4 promoter AID genomic regions occupied by Oct-4/Sox2 [6]. Depletion of SCC/XPC resulted in reduction of pluripotency in ESCs and reprogramming into iPSCs, implying that loss of the SCC/XPC complex decreased transcriptional integrity and the ability of pluripotency reestablishment. Thus SCC/XPC is required for the modulation of self-renewal and reprogramming efficiency $[6,12]$. SCC/XPC is also involved in the process of chromatin reorganization and alternation of the epigenetic landscape for iPSCs conversion [13]. 


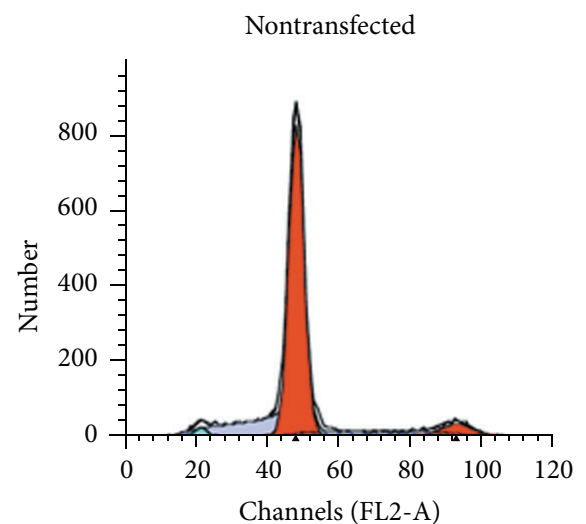

(a1)

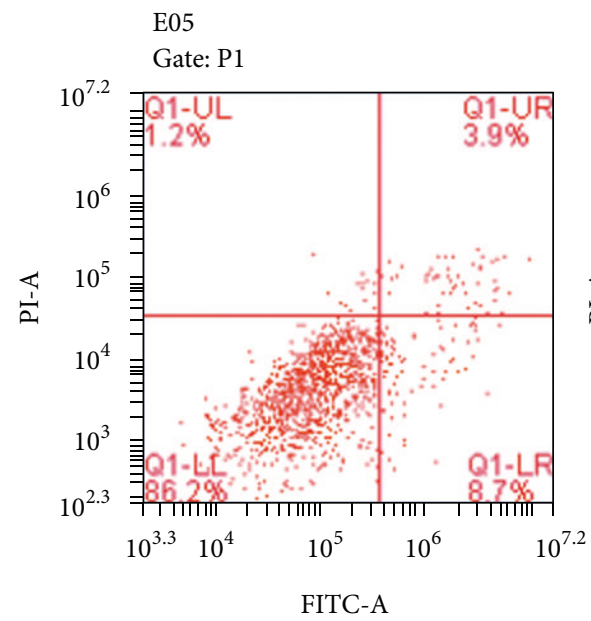

(a4)

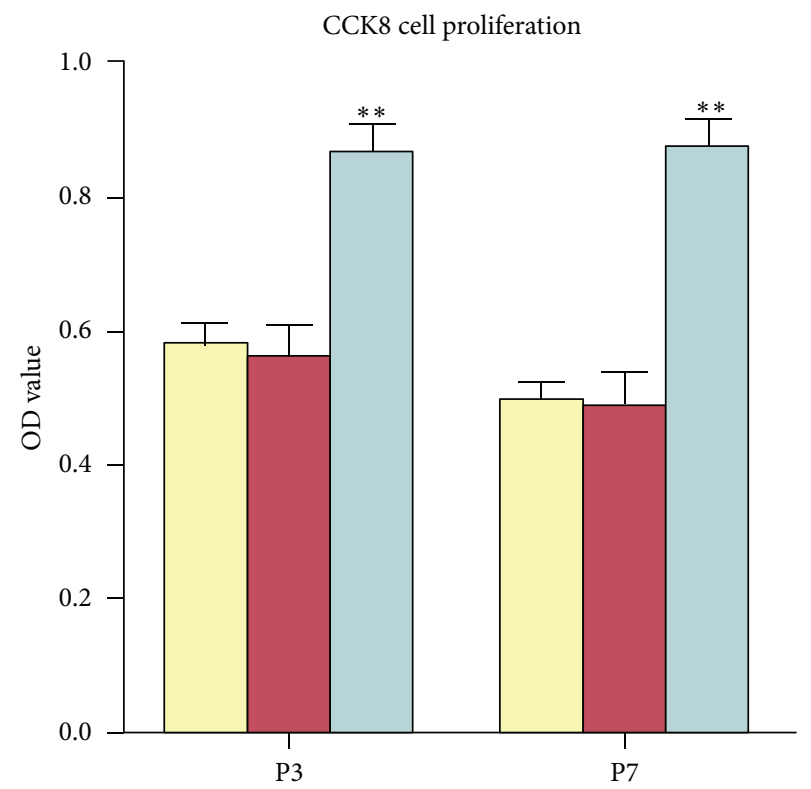

(b1)

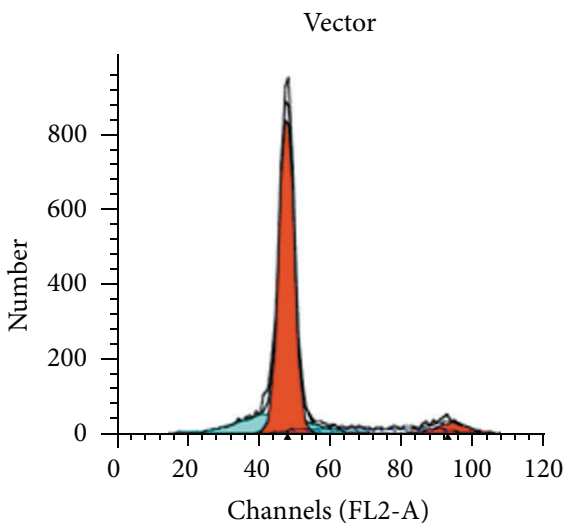

(a2)

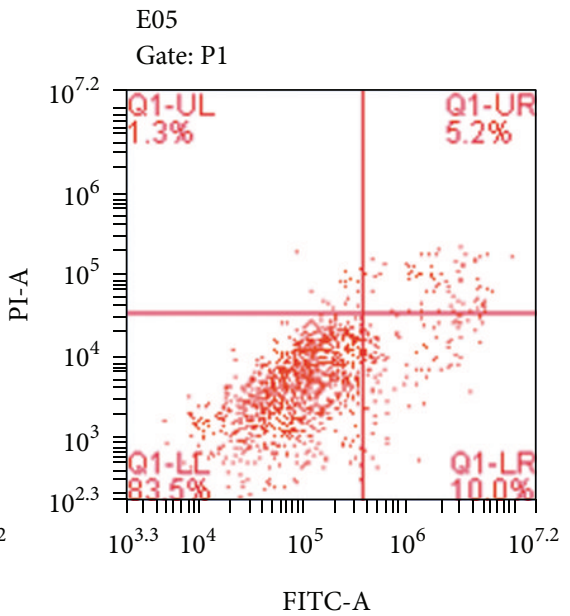

(a5)

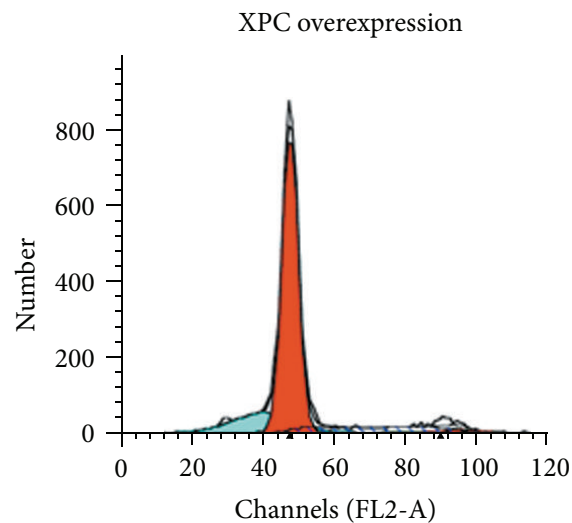

(a3)

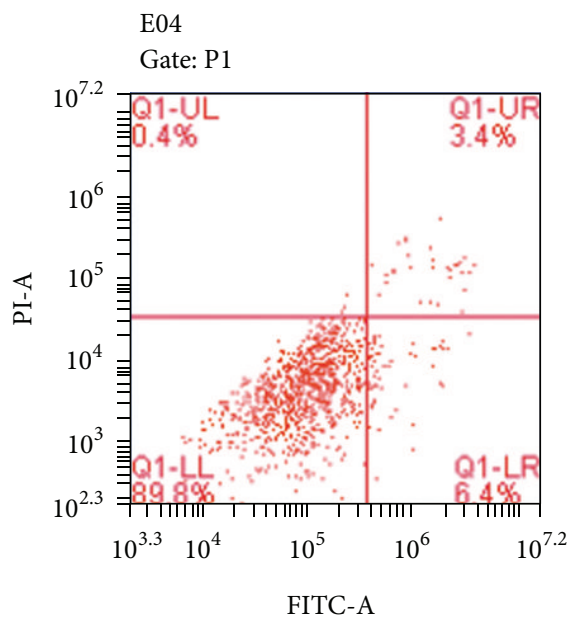

(a6)

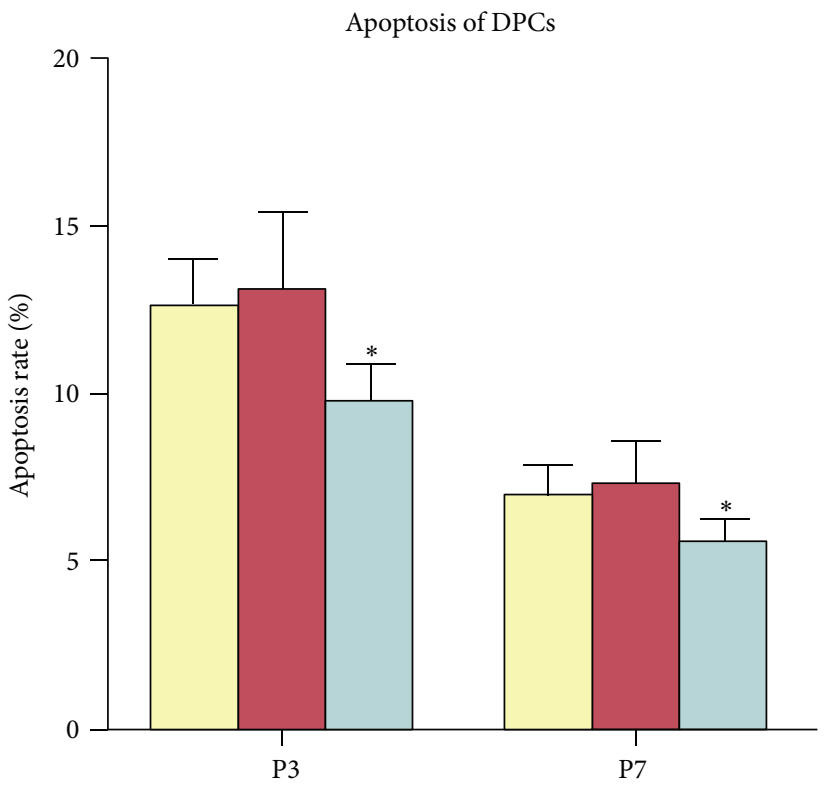

(b2)

Figure 3: Continued. 


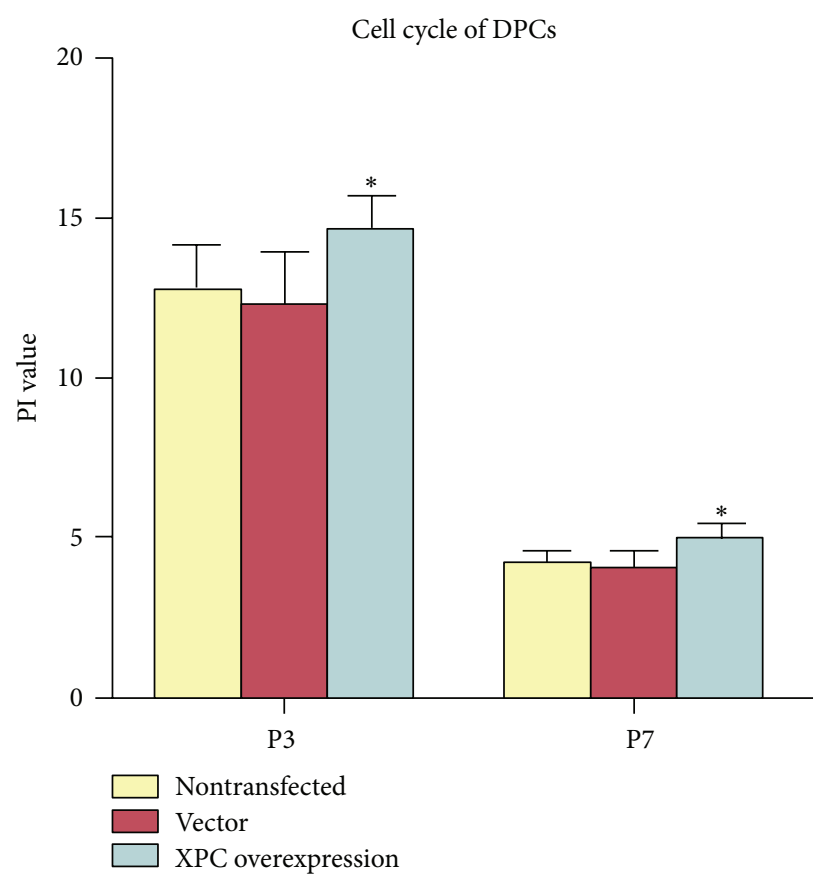

(b3)

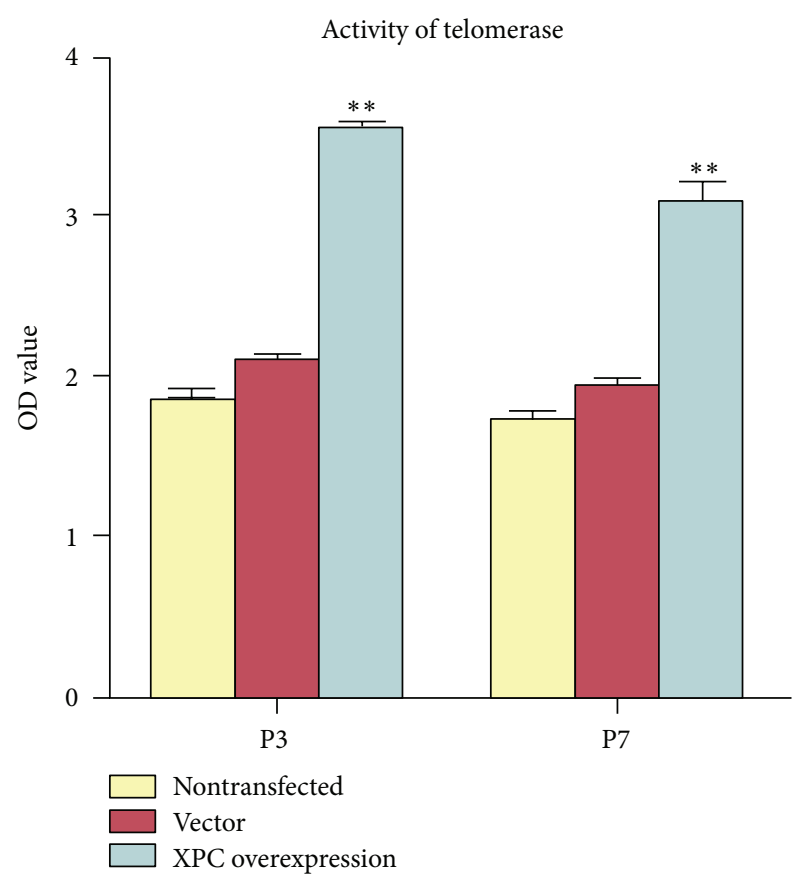

(b4)

FIGURE 3: Effect of XPC on cell proliferation and apoptosis and cell cycle and senescence of DPCs with in vitro culture. CCK8 revealed that cell proliferation of transfected DPCs at both P3 and P7 was significantly enhanced (b1, ${ }^{* *} p<0.001$ ). The result of FACS (a1-a6) demonstrated that the cell number of transfected DPCs at P3 and P7 in G0/G1 was significantly downregulated compared with the vector groups; DPCs were arrested in G2/M and S phase. The percentage of $\mathrm{PI}=(\mathrm{S}+\mathrm{G} 2 / \mathrm{M})$ value $\left(\mathrm{b} 3,{ }^{*} p<0.05\right)$ and telomerase activity $\left(\mathrm{b} 4,{ }^{* *} p<0.001\right)$ of transfected DPCs at both P3 and P7 was significantly upregulated, albeit the apoptosis rate was significantly downregulated (b2, $\left.{ }^{*} p<0.05\right)$.

$\mathrm{XPC}$, as one component of the NER, functions as a transcriptional coactivator independently of DNA repair through modifying chromatin. XPC is the essential element to recognize DNA distortions and recruit the basal transcription factors, like TFIIH [14]. XPC trimeric complex is required to activate the Oct-4/Sox 2 mediated transcription for the maintenance and reestablishment of ESCs specific gene expression $[6,14]$. Removal of the C-terminal region of XPC, including the interaction sites with $\operatorname{Rad} 23$ and Cetn2, resulted in alternations of the gene expression profile and pluripotency of ESCs [7]. Downregulation of XPC and RAD23B could significantly decrease Oct-4/Sox 2 dependent transcription of Nanog, indicating that SCC/XPC is closely associated with pluripotency [12]. XPC mainly binds to RAD23B, which stabilizes XPC and effectively binds XPC to DNA lesions. Simultaneous knock-down of XPC, RAD23B, and CETN2 resulted in decrease in expression of Oct-4 and self-renewal of ESCs [12]. XPC silencing resulted in decreased amount of CENTRIN2 expression, which affected both its centrosomal and nuclear localization. Thus XPC deficiency indirectly slows down cell division and lower DNA repair capacity, confirming the essential role XPC played in CENTRIN2's internalization into the nucleus of human cells, DNA repair, and cell survival [15].

In our previous study, we revealed that the mRNA and protein expression of Oct-4, Sox 2, and c-Myc in DPCs were firstly upregulated and peaked at passage 2 and then they were subsequently downregulated afterwards [8]. In the present study, real-time PCR and western blot indicated that XPC showed a similar expression pattern with Oct-4, Sox2, and $\mathrm{c}-\mathrm{Myc}$, suggesting that XPC might work cooperatively with Oct-4, Sox2, and c-Myc in the maintenance and reestablishment of pluripotency in DPCs with in vitro culture. Moreover, Oct-4, Sox2, and c-Myc significantly increased in DPCs with XPC overexpression. These results agreed with previous reports that XPC is required to activate the Oct-4/Sox2 mediated maintenance and reestablishment of pluripotency through interacting with downstream transcription factors $[6,12]$.

Prolonged in vitro culture has been reported to cause morphologic alternations and decrease expression of pluripotent markers and cell senescence $[16,17]$. Oncogenic stress, DNA damage and stress stimulation, and so forth are contributory factors to cell senescence. Accumulation of DNA deletions or mutations can result in aging and senescence [16]. DNA damage with genomic defect or mutation could stimulate oncogenes and inactivate tumor suppressor genes, causing accelerate aging, loss of homeostasis, and tumorigenesis [17]. DNA repair is essential to the homeostasis and genomic stability of all organisms [18]. Cell cycle arrest could prevent mutagenesis and DNA damage [19]. NER factors are related to cell cycle checkpoints, which control apoptosis, cell cycle arrest, and DNA repair and enhance genomic stability and cell survival [20]. It has been shown that oxidative DNA damage accumulation increased without presence of XPC. $\mathrm{XPC}$ is involved in the in vivo and in vitro initiation of 


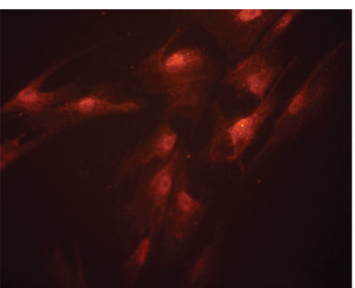

(a1)

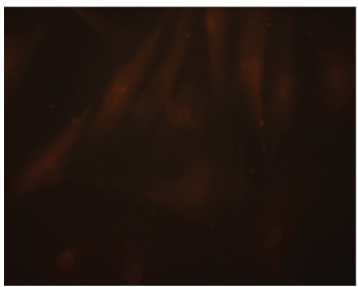

(a5)

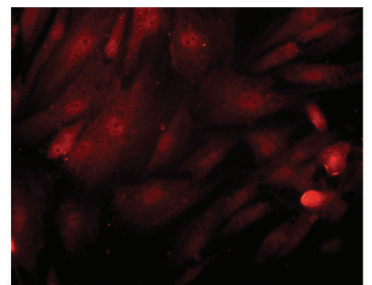

(a2)

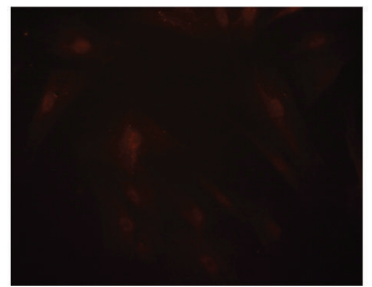

(a6)

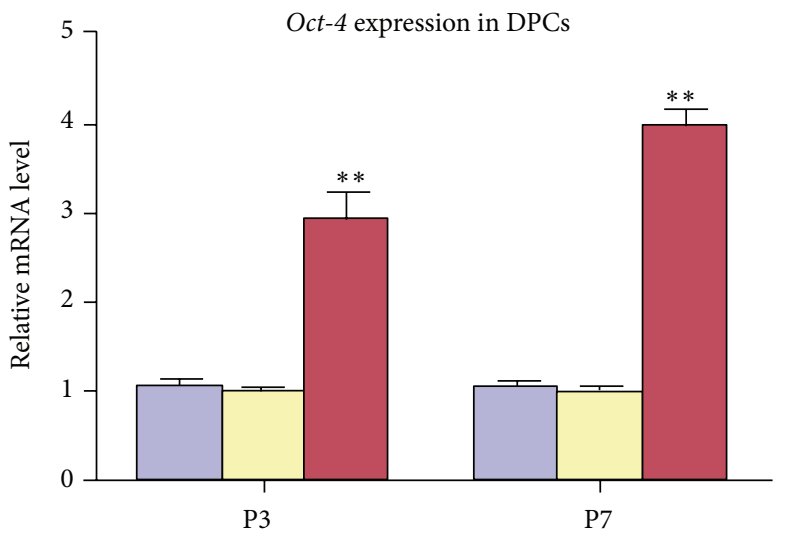

(b1)

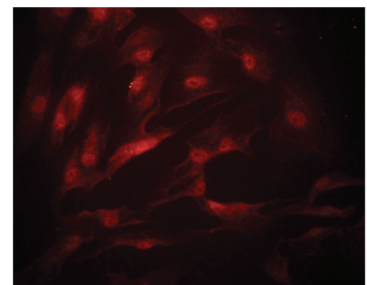

(a3)

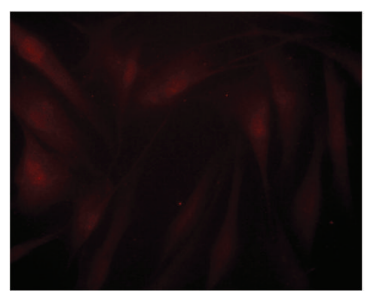

(a7)

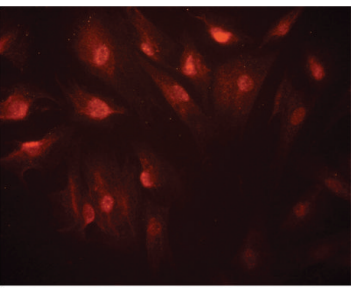

(a4)

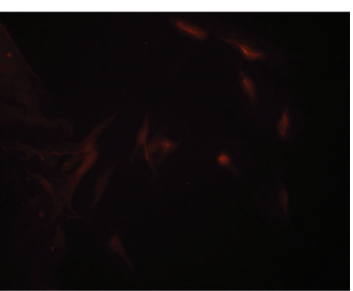

(a8)

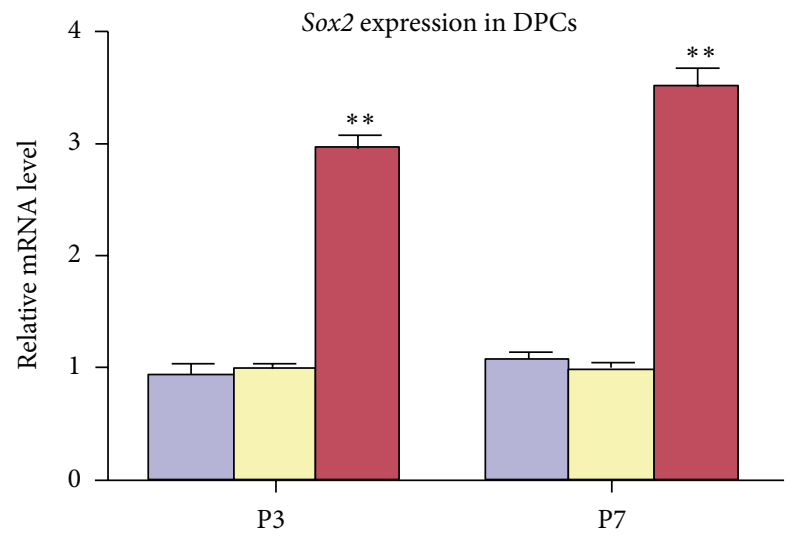

(b2)

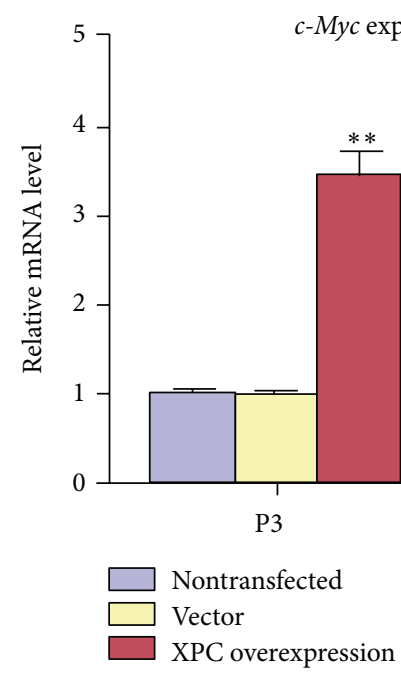

(b3)

FIGURE 4: Effect of XPC on the expression of Oct-4, Sox2, and c-Myc in DPCs at various passages. Immunofluorescent staining revealed that XPC (a1), Oct-4 (a2), Sox2 (a3), and c-Myc (a4) were strongly expressed in DPCs with XPC overexpression at P7, mainly located in nucleus and moderately expressed in cytoplasm, whereas XPC (a5), Oct-4 (a6), Sox2 (a7), and c-Myc (a8) were barely detected in DPCs at P7 without transfection. Real-time PCR indicated that the mRNA expression of XPC, Oct-4, Sox2, and $c-M y c$ was upregulated significantly in DPCs with XPC overexpression at both P3 and P7 compared with vector groups $\left({ }^{* *} p<0.001\right)$. There is no significant difference between nontransfected group and vector group $(p>0.05)$ (b1-b3). 


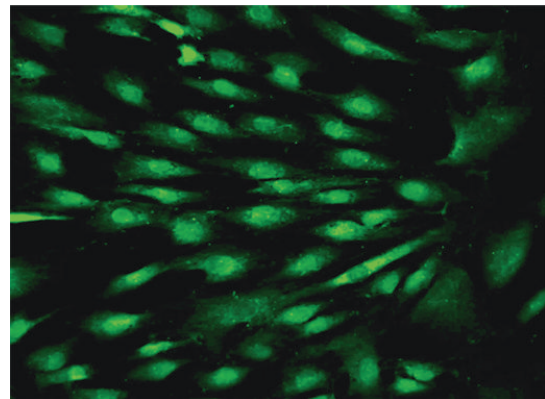

(a1)

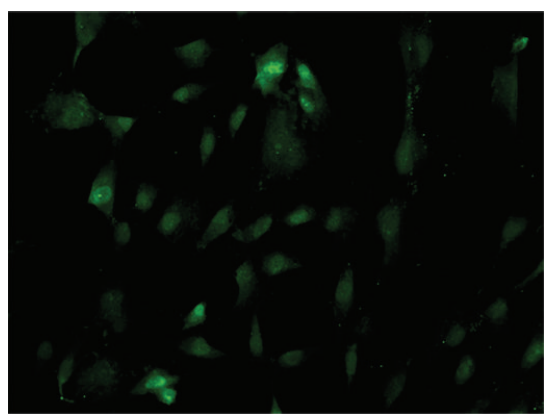

(a4)

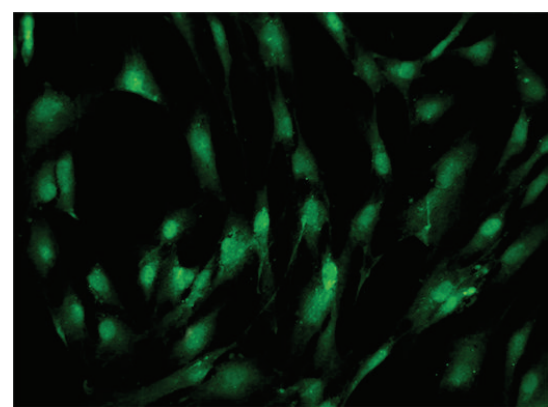

(a2)

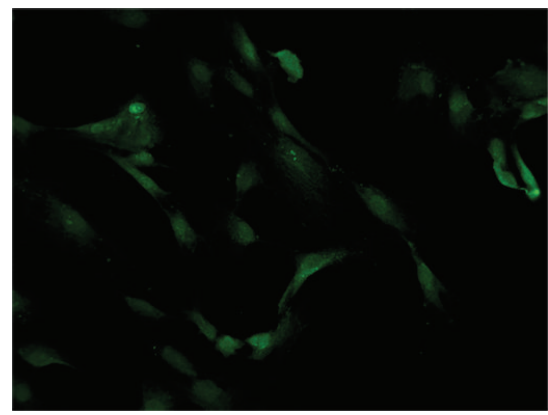

(a5)

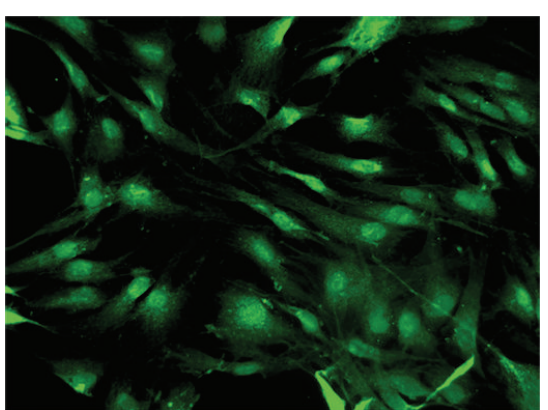

(a3)

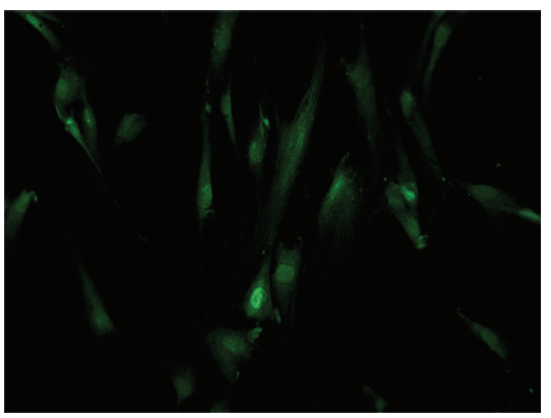

(a6)

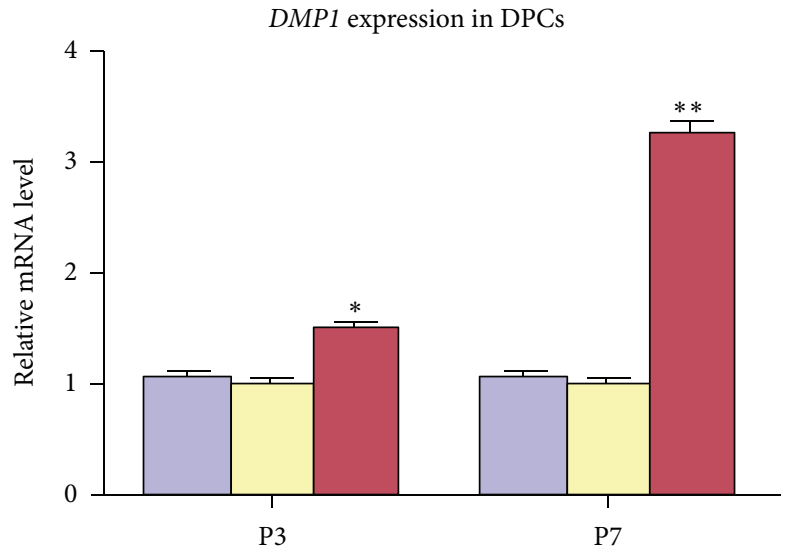

(b1)

PPAR $\gamma 2$ expression in DPCs

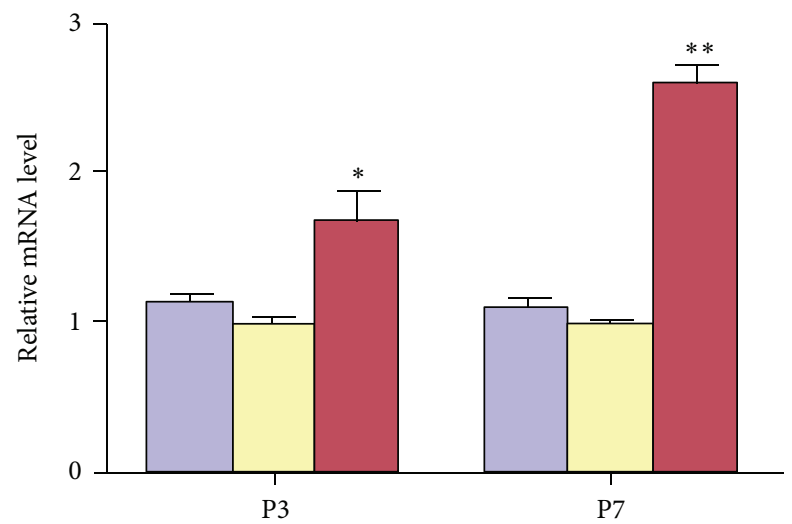

(b3)

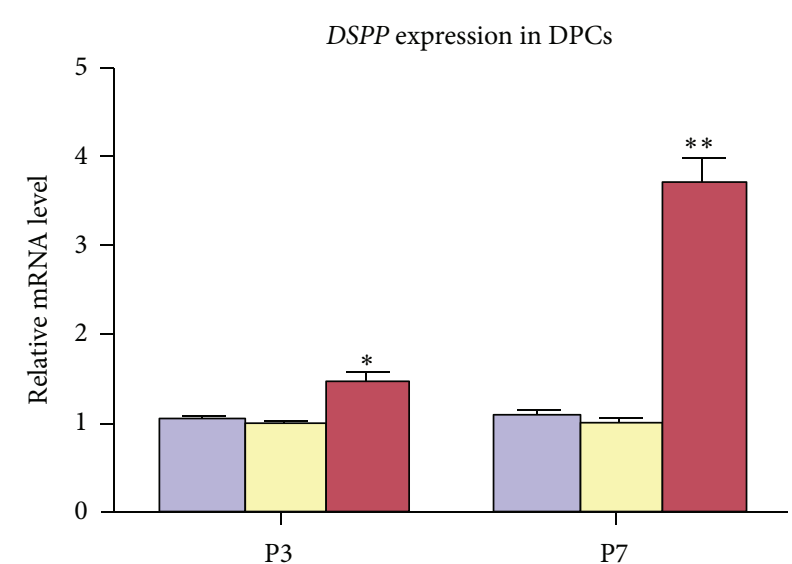

(b2)

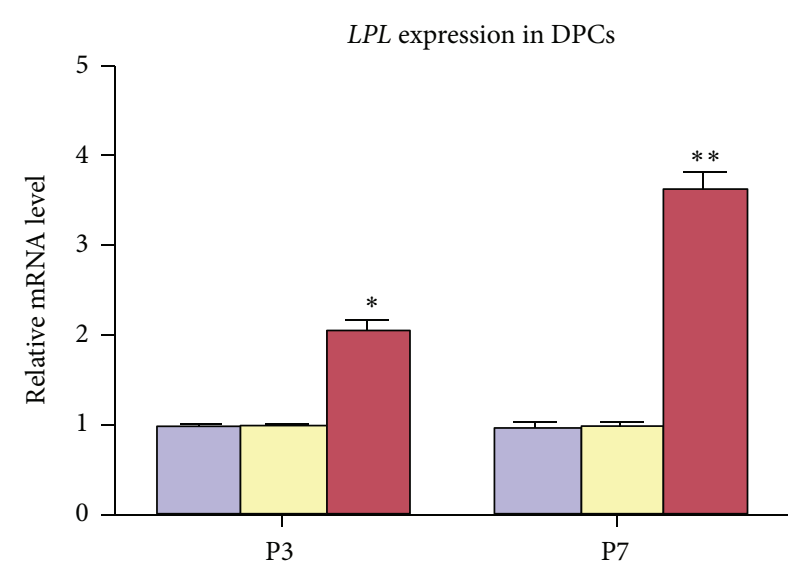

(b4)

FIgURE 5: Continued. 


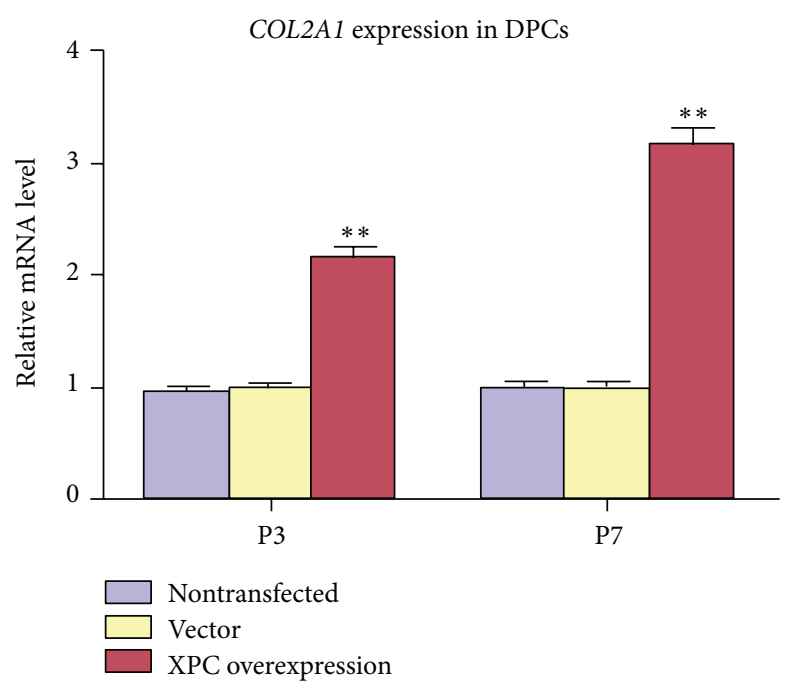

(b5)

FIGURE 5: Effect of XPC on the multilineage differentiation capability of DPCs after transfection. After $21 \mathrm{~d}$ of multilineage differentiation induction, immunofluorescent staining indicated that DSPP (a1), LPL (a2), and collagen type II (a3) were extensively expressed in DPCs at P7 with XPC overexpression, strongly expressed in the nucleus and moderately expressed in the cytoplasm of DPCs. However, DSPP (a4), LPL (a5), and collagen type II (a6) revealed weak expression in the nucleus and cytoplasm of DPCs at P7 without transfection. Real-time PCR indicated that mRNA expression level of odontogenic markers (DMP1, DSPP), adipogenic markers (PPAR $2, L P L)$, and chondrogenic markers (collagen type II) increased significantly in DPCs at P3 and P7 with XPC overexpression compared with vector groups (b1-b5).

DNA damage-induced biological process, including removal of oxidative DNA damage, redox homeostasis, and cell cycle regulation $[14,21]$. Persistent DNA damage without XPC resulted in sustaining telomere attrition; thus XPC is required to maintain telomere length, integrity, and stability. Failure of DNA repair and telomere shortening result in replicative senescence or cell death [22].

In the present study, significant upregulation of cell proliferation, telomerase activity, and multilineage differentiation was observed in DPCs with XPC overexpression, while apoptosis was restrained in DPCs with XPC overexpression. Moreover, we observed an increase in the percentage of G2/M and S phase cells in DPCs after XPC transfection. Considering that XPC plays dual roles in transcriptional coactivation and DNA repair to maintain pluripotency in ESCs $[6,12,15]$, the result from present study implied that XPC might modulate cell senescence and pluripotency through cell cycle regulation. It might be possible that XPC increases pluripotency of DPCs through elimination of damaged cells, subsequently reducing the risk of cell cycle reentry and acquisition of mutations. These results agreed with the previous reports and expanded our data regarding the effect of XPC on cell senescence and pluripotency in dental derived cells $[6,12,15,22]$.

\section{Conclusions}

Taken together, the present study demonstrated that pluripotency could be reachieved in DPCs at late passages with XPC overexpression through regulation of Oct-4/Sox $2 / \mathrm{c}$ Myc network; therefore XPC may play an essential role in the modulation of pluripotency and multilineage differentiation of DPCs via regulation of Oct-4/Sox2/c-Myc. The knowledge generated from this study will offer better understanding on the potential application of DPCs in dental tissue engineering and improving the method to induce pluripotent cells.

\section{Competing Interests}

The authors declare that they have no competing interests.

\section{Authors' Contributions}

Lu Liu and Zhengjun Peng contributed equally as first coauthors to this study.

\section{Acknowledgments}

This study was supported by National Natural Science Foundation of China (81371133/81400499) and Guangdong Provincial Department of Science and Technology (2015A020212004).

\section{References}

[1] Y. Zheng, M. Chen, L. He et al., "Mesenchymal dental pulp cells attenuate dentin resorption in homeostasis," Journal of Dental Research, vol. 94, no. 6, pp. 821-827, 2015.

[2] G. Asatrian, D. Pham, W. R. Hardy, A. W. James, and B. Peault, "Stem cell technology for bone regeneration: current status and potential applications," Stem Cells and Cloning: Advances and Applications, vol. 8, pp. 39-48, 2015.

[3] L. Liu, J. Ling, X. Wei, L. Wu, and Y. Xiao, "Stem cell regulatory gene expression in human adult dental pulp and periodontal 
ligament cells undergoing odontogenic/osteogenic differentiation," Journal of Endodontics, vol. 35, no. 10, pp. 1368-1376, 2009.

[4] K. Takahashi and S. Yamanaka, "Induction of pluripotent stem cells from mouse embryonic and adult fibroblast cultures by defined factors," Cell, vol. 126, no. 4, pp. 663-676, 2006.

[5] Y. X. Fan, C. H. Gu, Y. L. Zhang et al., "Oct4 and Sox2 overexpression improves the proliferation and differentiation of bone mesenchymal stem cells in Xiaomeishan porcine," Genetics and Molecular Research, vol. 12, no. 4, pp. 6067-6079, 2013.

[6] Y. W. Fong, C. Inouye, T. Yamaguchi, C. Cattoglio, I. Grubisic, and R. Tjian, "A DNA repair complex functions as an Oct4/Sox2 coactivator in embryonic stem cells," Cell, vol. 147, no. 1, pp. 120131, 2011.

[7] S. Ito, M. Yamane, S. Ohtsuka, and H. Niwa, "The C-terminal region of $\mathrm{Xpc}$ is dispensable for the transcriptional activity of Oct3/4 in mouse embryonic stem cells," FEBS Letters, vol. 588, no. 7, pp. 1128-1135, 2014.

[8] L. Liu, X. Wei, J. Ling, L. Wu, and Y. Xiao, "Expression pattern of Oct-4, sox2, and c-Myc in the primary culture of human dental pulp derived cells," Journal of Endodontics, vol. 37, no. 4, pp. 466-472, 2011.

[9] L. Liu, L. Wu, X. Wei, and J. Ling, "Induced overexpression of Oct4A in human dental pulp cells enhances pluripotency and multilineage differentiation capability," Stem Cells and Development, vol. 24, no. 8, pp. 962-972, 2015.

[10] S. Schoeftner, M. Scarola, E. Comisso, C. Schneider, and R. Benetti, "An Oct4-pRb axis, controlled by miR-335, integrates stem cell self-renewal and cell cycle control," STEM CELLS, vol. 31, no. 4, pp. 717-728, 2013.

[11] C. Sawan, H. Hernandez-Vargas, R. Murr et al., "Histone acetyltransferase cofactor Trrap maintains self-renewal and restricts differentiation of embryonic stem cells," STEM CELLS, vol. 31, no. 5, pp. 979-991, 2013.

[12] J.-P. Etchegaray and R. Mostoslavsky, "eNERgizing pluripotent gene transcription," Cell Stem Cell, vol. 9, no. 4, pp. 285-286, 2011.

[13] N. Le May, D. Mota-Fernandes, R. Vélez-Cruz, I. Iltis, D. Biard, and J. M. Egly, "NER factors are recruited to active promoters and facilitate chromatin modification for transcription in the absence of exogenous genotoxic attack," Molecular Cell, vol. 38, no. 1, pp. 54-66, 2010.

[14] S. M. Shell, E. K. Hawkins, M.-S. Tsai, A. S. Hlaing, C. J. Rizzo, and W. J. Chazin, "Xeroderma pigmentosum complementation group C protein (XPC) serves as a general sensor of damaged DNA," DNA Repair, vol. 12, no. 11, pp. 947-953, 2013.

[15] E. Renaud, L. Miccoli, N. Zacal et al., "Differential contribution of XPC, RAD23A, RAD23B and CENTRIN 2 to the UVresponse in human cells," DNA Repair, vol. 10, no. 8, pp. 835847, 2011.

[16] A. Kowald and T. B. L. Kirkwood, "Mitochondrial mutations and aging: random drift is insufficient to explain the accumulation of mitochondrial deletion mutants in short-lived animals," Aging Cell, vol. 12, no. 4, pp. 728-731, 2013.

[17] M. D. Burkhalter, K. L. Rudolph, and T. Sperka, "Genome instability of ageing stem cells-induction and defence mechanisms," Ageing Research Reviews, vol. 23, pp. 29-36, 2015.

[18] A. Bravard, F. Auvré, D. Fantini et al., "The prion protein is critical for DNA repair and cell survival after genotoxic stress," Nucleic Acids Research, vol. 43, no. 2, pp. 904-916, 2015.

[19] D. Speidel, "The role of DNA damage responses in p53 biology," Archives of Toxicology, vol. 89, no. 4, pp. 501-517, 2015.
[20] M. A. Ermolaeva, A. Segref, A. Dakhovnik et al., "DNA damage in germ cells induces an innate immune response that triggers systemic stress resistance," Nature, vol. 501, no. 7467, pp. 416420, 2013.

[21] H. Menoni, J. H. J. Hoeijmakers, and W. Vermeulen, "Nucleotide excision repair-initiating proteins bind to oxidative DNA lesions in vivo," Journal of Cell Biology, vol. 199, no. 7, pp. 1037-1046, 2012.

[22] G. J. Stout and M. A. Blasco, "Telomere length and telomerase activity impact the UV sensitivity syndrome xeroderma pigmentosum C," Cancer Research, vol. 73, no. 6, pp. 1844-1854, 2013. 

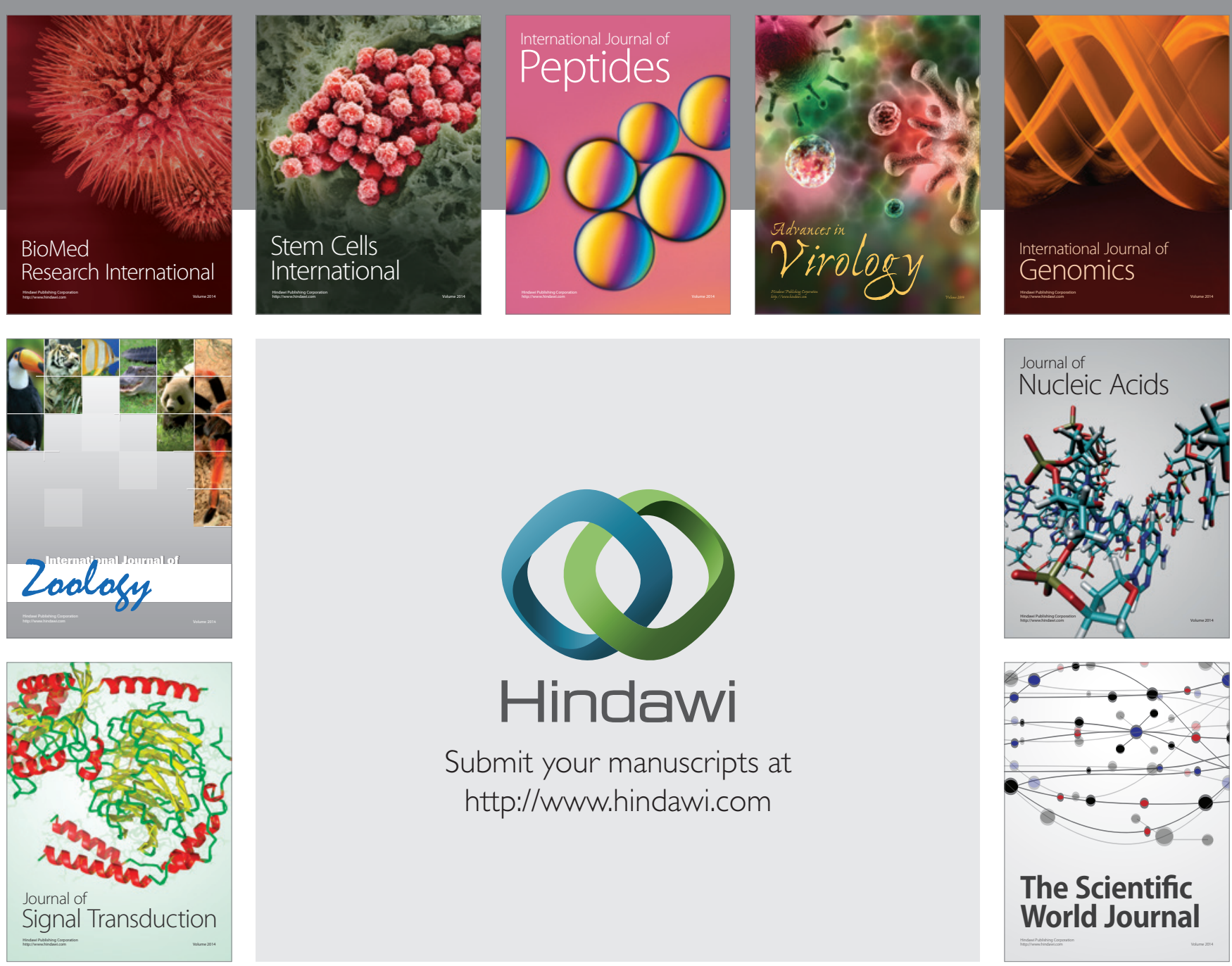

Submit your manuscripts at

http://www.hindawi.com
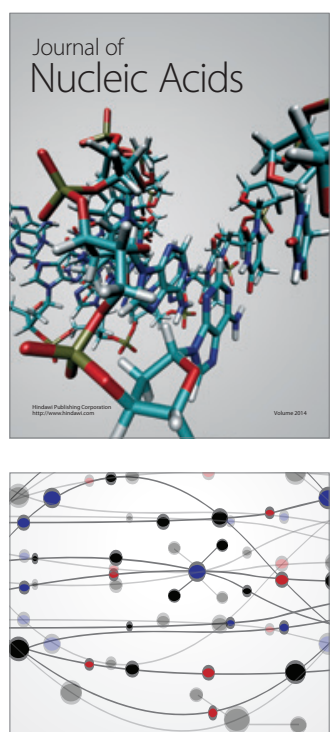

The Scientific World Journal
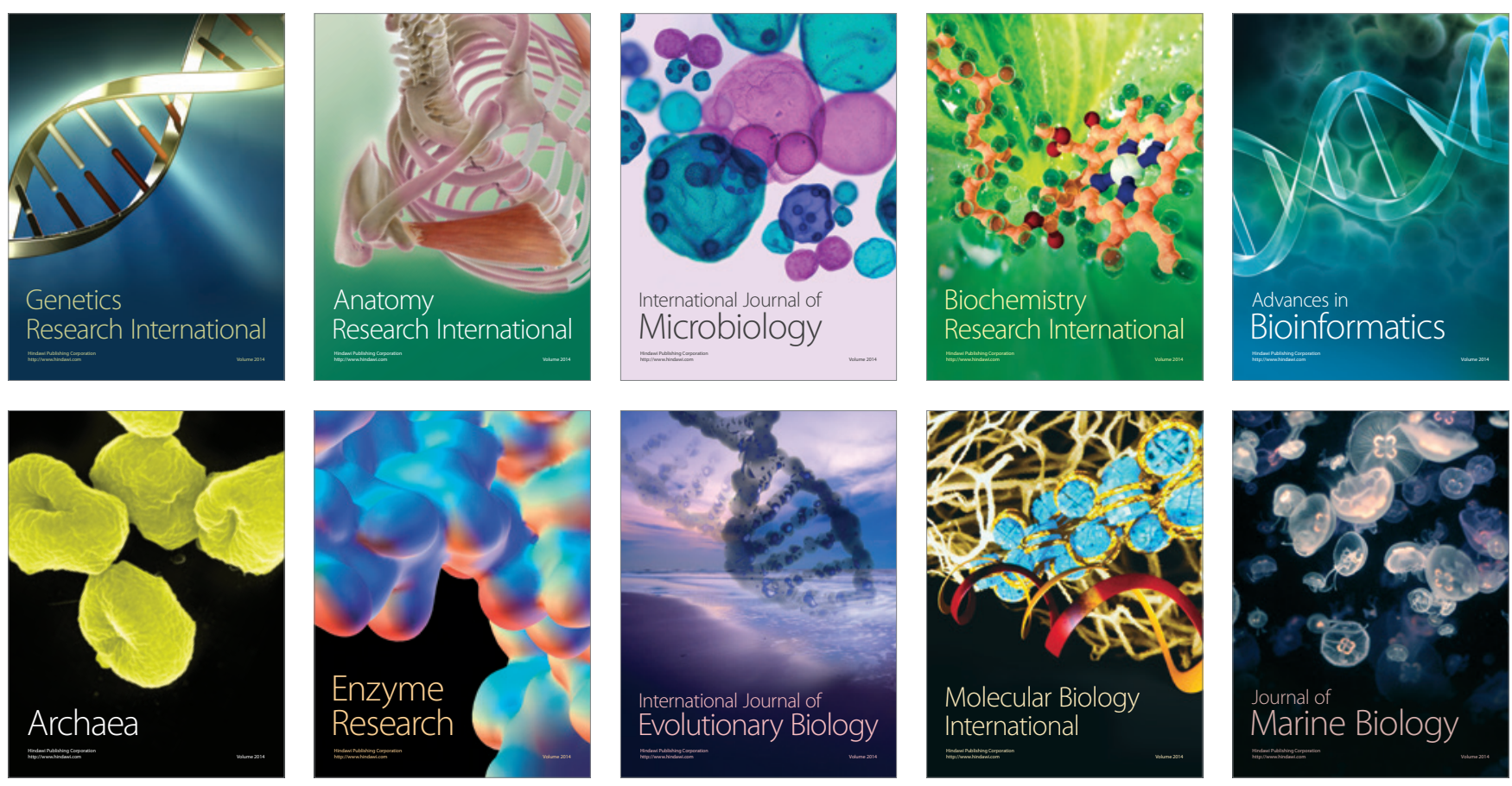